\title{
Reflexiones sobre oportunidades de intervención en tabaquismo en Latinoamérica
}

\author{
Gustavo Zabert, MC,, José Miguel Chatkin, MC Neumól, (2) Guadalupe Ponciano-Rodríguez, MC.(3)
}

\author{
Zabert G, Chatkin JM, Ponciano-Rodríguez G. \\ Reflexiones sobre oportunidades \\ de intervención en tabaquismo en Latinoamérica. \\ Salud Publica Mex 2010;52 supl 2:S283-S287.
}

\begin{abstract}
Resumen
El tabaquismo es un grave problema de salud pública en Latinoamérica, cuyos países aún muestran en su estructura poblacional una amplia base piramidal formada por grupos etarios de jóvenes que han sido el blanco primordial de la industria tabacalera. Latinoamérica requiere urgentemente de estrategias de prevención y acciones sanitarias para modificar las consecuencias de fumar sobre la salud de la población a través de la cesación del tabaquismo. El tratamiento de la adicción a la nicotina requiere estrategias de intervención eficaces para disminuir la prevalencia de fumadores y evitar su recaída, brindándoles herramientas para el mantenimiento de la abstinencia. En la actualidad, poblaciones pobres y vulnerables ( $44 \%$ de la población latinoaméricana) están condenadas a sufrir las consecuencias sanitarias de esta enfermedad, ya que son las que presentan un mayor consumo de tabaco y las que pueden acceder menos a las intervenciones sanitarias. El Convenio Marco para el Control del Tabaco (CMCT) y el MPOWER, ambos estrategias de la Organización Mundial de la Salud para la lucha contra el tabaco, definen claras líneas de acción pero sus limitaciones están definidas por los recursos que se asignan para las mismas en cada país. La comunidad científica y técnica abocada a la lucha contra este flagelo debe considerar y poner en marcha nuevas perspectivas para lograr colocar en la agenda política la necesidad de obtener y asignar más recursos económicos y humanos para disminuir el consumo del tabaco en todos los grupos de la población. En la lucha contra el tabaco, este es el momento indicado para considerar primordialmente aspectos de equidad y justicia social en salud.
\end{abstract}

Palabras clave: tabaco; cesación del tabaquismo; intervención; guías de tratamiento; CMCT; MPOWER
Zabert G, Chatkin JM, Ponciano-Rodríguez G.

Reflections on smoking intervention opportunities

In Latin america.

Salud Publica Mex 2010;52 suppl 2:S283-S287.

\begin{abstract}
The tobacco epidemic is a huge public health problem affecting all Latin American countries. These countries have the characteristic of a wide base of the pyramidal population structure, with many young people who have been natural targets for the tobacco industry. Therefore in this region there is an urgent need to establish prevention and treatment strategies capable to reduce incidence and prevalence of tobacco smoking in the population and as a consequence modify the health effects of tobacco consumption. Treating nicotine addiction requires intervention strategies really effective to reduce the number of current smokers as well as giving them tools to avoid relapse and maintain abstinence. Currently poor and vulnerable groups, which represent $44 \%$ of the total population in Latin America, are the most susceptible to suffer the health consequences of smoking since they show the highest prevalence rates and have little or no access to health services. The Framework Convention on Tobacco Control (FCTC) and MPOWER, both strategies proposed by the World Health Organization against tobacco, define clear goals to achieve their purposes, nevertheless they are limited by the economic and human resources assigned by each country. The scientific community involved in the fight against tobacco must think about new ways to place this issue in the political agenda so there will be more opportunities to increase the resources and therefore to reduce tobacco consumption in all the groups of the population. In the fight against tobacco, this is the right time to consider primarily issues related to equity and social justice.
\end{abstract}

Key words: tobbacco; smoking cessation; intervention; guidelines of treatment; FCTC; MPOWER

(I) Escuela de Medicina, UNComahue. Neuquen,Argentina.

(2) Facultad de Medicina. Porto Alegre, Brasil.

(3) Clínica para el Tratamiento del Tabaquismo. Facultad de Medicina, UNAM. México.

Fecha de recibido: 22 de marzo de 2010 • Fecha de aceptado: 29 de junio de 2010 Solicitud de sobretiros: Dra. Guadalupe Ponciano Rodríguez. Departamento de Salud Pública. Facultad de Medicina, UNAM. Circuito Interior de Ciudad Universitaria, col. Coyoacán. México 045I0, DF México.

Correo electrónico:ponciano@servidor.unam.mx,sinfumar@mexico.com 
Las pequeñas oportunidades son el principio de las grandes empresas.

Demóstenes.

E tabaco ocasiona actualmente 6 millones de muertes anuales, de las cuales 845000 ocurren en Latinoamérica, ${ }^{1}$ sin embargo, para 2030 se estima que las muertes superarán los 8 millones $^{2}$ y 8 de cada 10 fallecimientos ocurrirán en los países en vías de desarrollo. El tabaco es la única conducta o producto de consumo legal que causa la muerte a 1 de cada 2 consumidores, sus víctimas superan a las guerras, las catástrofes naturales, el VIHSIDA y las drogas, en su conjunto. Ninguna metáfora describe mejor al tabaquismo que la del elefante dentro de una habitación, ${ }^{3}$ el cual por más que se intente no puede ser disimulado y ha llegado el momento en que tenemos que hablar de él.

La forma más rápida y efectiva de evitar estas funestas predicciones es lograr que los que ahora son fumadores abandonen el consumo, ${ }^{4}$ estrategia que se ha demostrado puede ser aún más efectiva que los esfuerzos puestos en la prevención para reducir en igual medida la prevalencia. Para lograr que los fumadores actuales alcancen la abstinencia es necesario desarrollar estrategias para desalentar el consumo y estimular los intentos para dejar de fumar, pero además, que cada tentativa sea más efectiva. La principal limitante para optimizar las acciones son los recursos económicos y humanos disponibles y que los gobiernos efectivamente los asignen para la lucha contra el tabaquismo. En América Latina, los médicos y el personal del área de la salud debemos convencer a las clases dirigentes de la magnitud del problema y a las posibles soluciones, para lo cual es necesario expresarlas en términos de eficacia y costo-eficacia, para que así puedan ser útiles en la toma de decisiones políticas.

\section{Dos miradas y un objetivo}

Si bien el objetivo pareciera ser claro e inequívoco, aumentar las tasas de abstinencia de la población fumadora a la brevedad para evitar las predicciones de las consecuencias futuras de enfermedad y muerte sobre este grupo, existen dos diferentes miradas sobre cómo lograr dicho propósito que por momentos parecieran antagónicas.

Las corrientes de salud pública proponen hacer el mayor esfuerzo en políticas públicas, ${ }^{5}$ como aumento de impuestos, restricción del consumo en sitios públicos, campañas de promoción de la cesación, advertencias sanitarias y pictogramas en las cajetillas, etcétera. En otro punto de vista se posicionan los médicos que se dedican a la atención de los pacientes adictos al tabaco, a quienes podríamos llamar "clínicos tabacólogos", los cuales proponen hacer un mayor énfasis en las estrategias de cesación, ${ }^{6}$ con herramientas que inciden sobre la conducta del individuo, a través de intervenciones de tipo individual o grupal, apoyadas con tratamiento farmacológico para la reducción de la intensidad y de la duración del síndrome de abstinencia de nicotina y de la urgencia por fumar. ${ }^{78}$ Sin embargo, es reconocida la escasa capacitación y entrenamiento de los equipos de salud para realizar esta tarea, a pesar de que en países desarrollados se ha presentado una reducción en la prevalencia de tabaquismo en el personal de salud, situación que contrasta con la de países en desarrollo, donde las tasas de tabaquismo en médicos y personal de salud pueden llegar incluso a superar las de la población general. $^{9-12}$

El $\mathrm{CMCT}^{13}$ y el documento MPOWER ${ }^{14}$ postulan que ambas acciones estratégicas son propuestas complementarias y así debieran ser reconocidas por la comunidad que lucha contra el tabaco, sin embargo, esto no funciona así en la vida real. Tanto en América Latina como en el resto del mundo, con alguna frecuencia adeptos de ambas corrientes se encuentran en espacios comunes estableciendo una confrontación por evidencias científicas, recursos y oportunidades que favorezcan una u otra perspectiva y distrayéndose de la lucha común contra el verdadero enemigo y a favor de la población a la que se deben. Éste es el camino para comenzar a perder la batalla y desaprovechar las oportunidades que se están abriendo en los últimos años para la auténtica empresa que es modificar las predicciones sobre las consecuencias del tabaco en la salud de la población. La comunidad que lucha contra el tabaco debe reconocer que es un problema de naturaleza compleja y que por ende requiere de la interacción plena de todos los actores fieles al accionar.

Más allá de esto, y con el único fin de acercar las visiones, los seguidores de una y otra tendencia deben reconocer que trabajan en dos niveles de análisis diferentes pero complementarios: los "sanitaristas" proponen acciones de intervención comunitarias y los "clínicos tabacólogos" tienen la mirada puesta en el ámbito individual.

Son escasos a la fecha los ejemplos que, como en Uruguay, se imbrican y desarrollan acciones conjuntas que demuestran la clara disposición de los tomadores de decisiones de mantener el problema en la agenda política con objetivos definidos y asignando los recursos necesarios para definirlos como una política de Estado.

Los seguidores de la corriente de salud pública centran las estrategias en el ámbito comunitario que se pueden resumir en acciones que apuntan a la reducción 
de demanda y de oferta de los productos de tabaco. ${ }^{13}$ Este accionar requiere, en la mayoría de sus actuaciones, de un grupo técnico multidisciplinario relativamente pequeño y altamente entrenado, con recursos y respaldo político para avanzar en los objetivos planteados. La reducción del consumo y de la prevalencia resulta como consecuencia de las acciones puestas en marcha y ejecución de medidas de vigilancia. Para un Estado ejecutor implica asignar recursos que son fácilmente presupuestables, previsibles y acotados, sin mayor incertidumbre en los recursos a asignar. Por lo general deben ser acompañadas de la difusión y la promoción de las acciones implementadas, y que pueden ser exageradas por la propaganda, hechos que con frecuencia son vistos como convenientes y provechosos para los cuadros políticos.

En contraste, los médicos asistenciales y sus líderes de opinión que patrocinan la corriente que propone incentivar las estrategias de intervención para la cesación de fumar tal como se plantea en el artículo 14 del $\mathrm{CMCT}^{13,15,16}$ alegan que este accionar impactaría rápidamente en la prevalencia y reducción del consumo en relación directamente proporcional con la magnitud de las intervenciones y de la efectividad de las mismas. Los recursos necesarios, entiéndase sólo para la capacitación del recurso humano, ${ }^{15-16}$ infraestructura y de los fármacos de probada eficacia, ${ }^{17-19}$ dependerán de la estrategia planteada pero también de la demanda. Es posible entonces que, a mayor éxito de los programas, mayor expectativa de la población, y por ende, mayor demanda, lo que podría derivar en una escalada de asignación de recursos para este fin. Este escenario propone incertidumbre e imprevisibilidad presupuestaria, eventualidad que preocupa a los tomadores de decisiones de políticas públicas y a los administradores de recursos en salud. Por ello, la estrategia MPOWER se anticipa a esta eventualidad mencionando que las intervenciones para el tratamiento del tabaquismo tienen un impacto mayor en la salud y son mucho más costo-eficaces que la mayoría de las actividades realizadas en el marco de los sistemas de atención a la salud. ${ }^{14}$ Este dilema se amplifica cuando los periodos de gestión de los actores políticos se acortan, ya que perciben que durante su periodo deberán mostrar resultados de eficacia y eficiencia de las estrategias implementadas quizás percibiendo de antemano que la balanza de costo-beneficio de las mismas se obtiene no a corto, sino a mediano y largo plazo.

\section{Equidad y justicia social en tabaquismo}

Se debe tomar en cuenta que hay argumentos en el mismo $\mathrm{CMCT}^{13}$ que exigen superar este nivel de análisis, dado que ratifica lo propuesto en el preámbulo de la Constitución de la OMS, en el que se establece como objetivo primordial el goce del grado máximo de salud que se pueda lograr como uno de los derechos fundamentales de todo ser humano sin distinción de raza, religión, ideología política o condición económica o social y se confirma por las declaraciones de la directora actual de este organismo, la Dra. Margaret Chan, al decir que la "máxima preocupación debe centrarse siempre en los grupos desfavorecidos y vulnerables. Estos grupos parecen a menudo ocultos, pues viven en zonas rurales remotas o en barrios marginales y apenas tienen voz política".

En cada rincón de América Latina la pobreza avanza, y por más que haya planes sociales que apunten a disminuir las diferencias, las desigualdades económicas y sociales que existen en la región son cada vez más pronunciadas y notorias. Según datos del Banco Interamericano de Desarrollo (BID), $44 \%$ de la población de América Latina es pobre y $40 \%$ de ellos son indigentes que viven con menos de un dólar diario. De acuerdo con un informe de la Comisión Económica para América Latina y el Caribe (CEPAL), Latinoamérica es la región más desigual del mundo. Sólo 7 de los 18 países latinoamericanos analizados (Cuba no fue incluida en el estudio) estarían en condiciones de reducir en $50 \%$ la extrema pobreza antes de 2015. Éstos son Argentina, Chile, Colombia, Honduras, Panamá, República Dominicana y Uruguay. ${ }^{20}$

Siguiendo con este estudio, Brasil, Costa Rica, El Salvador, Guatemala, México y Nicaragua también disminuirán la extrema pobreza, pero a un ritmo más lento. Los casos más graves son Bolivia, Ecuador, Paraguay, Perú y Venezuela, donde los índices de indigencia tenderían a aumentar por efecto combinado de la caída en los ingresos de los estratos más desfavorecidos y por aumentos de la brecha de desigualdad. ${ }^{20}$

Es entonces que la justicia social, en términos de equidad en salud y la sensibilidad ante la necesidad, surge como necesaria en la programación e implementación de estrategias de cesación del tabaquismo. Con el primer concepto, que ya fue recientemente desarrollado en otra publicación, ${ }^{21}$ se plantea que es necesario proponer una definición operacional de equidad para el acceso y la distribución de recursos. Esta definición reclamaría una mirada diferente para las poblaciones de menores recursos y desprotegidas socialmente, que surgen como los grupos de mayor prevalencia de consumo de tabaco, alcohol y otras drogas ${ }^{22}$ y que tienen menor acceso a los servicios de tratamiento, ya sea para la cesación del tabaquismo como para las enfermedades asociadas.

Por otro lado, el concepto de sensibilidad ante la necesidad, que pretende resumir la efectiva respuesta a las expectativas de la población, debe reconocer que 
al tratar con adicciones sus individuos estarán profundamente influenciados por la dependencia ${ }^{23}$ y exige una perspectiva diferente al momento de realizar definiciones operativas de las acciones de intervención y los problemas de salud derivados. La efectividad que muestran nuevas estrategias ${ }^{17-19}$ y las experiencias aún sin publicar del Fondo de Recursos de Uruguay dan soporte a las nuevas tendencias que apuntan a modificar la motivación para abandonar el consumo. ${ }^{24}$

Es importante enfatizar que estudios realizados en América Latina demuestran que los grupos social y económicamente vulnerables de la población son además aquellos cuyos hogares se ven más afectados por el gasto en adquisición de tabaco, el cual compite con la obtención de satisfactores básicos como alimentos y educación, por lo que representa una verdadera carga económica. ${ }^{22,25}$

Sin embargo, en la mayoría de los países, aun en los que se predice el mayor impacto sanitario, los programas sanitarios y las acciones de intervenciones para la cesación del consumo de tabaco no figuran en las prioridades sanitarias tal como lo demuestra un estudio realizado entre expertos de 36 países, ${ }^{24}$ donde se destaca que menos de la mitad cuenta con una política oficial de tratamiento, un tercio paga por tratamientos intensivos y sólo 1 de cada 5 cuenta con un programa nacional de tratamiento. Profundizando el modelo de tabaco y pobreza, al agrupar a los países por nivel de ingreso, se observa que los de ingreso alto son los que tienen una mayor disponibilidad de recursos de manera que sólo 5\% de la población mundial viviendo en los países más ricos tiene acceso a los tratamientos para dejar de fumar. Para más evidencias, en el momento de realizar la encuesta, únicamente dos países ofrecían tratamiento sin cargo para los fumadores, lo cual ratifica un escenario de gran inequidad para las poblaciones más desprotegidas en Latinoamérica. ${ }^{24}$

En contraste, se ha demostrado que cuando se aplican modelos que combinan varias estrategias como incremento de impuestos, políticas de espacios libres de humo de tabaco, campañas masivas en los medios de comunicación, mensajes precautorios y limitación a los jóvenes del acceso a los productos de tabaco, tomando en consideración las características demográficas de la población, se obtiene una medición que sugiere una reducción del consumo de tabaco, infiriendo que se acompaña de caída en la prevalencia del tabaquismo. ${ }^{26}$ Desafortunadamente, en estos modelos no se demuestra el impacto individual y se esgrime como argumento que confronta a los programas de intervención para fumadores.

\section{Momentos oportunos}

Es momento de pensar que nos encontramos ante una encrucijada que podría ser única y el comienzo de un cambio para Latinoamérica: una gran mayoría de países latinoamericanos ha ratificado el $\mathrm{CMCT}^{27}$ entonces, se cuenta ya con una base legal de herramientas para definir las acciones comunitarias. El gran ausente son las acciones de intervención individual, establecidas en el Artículo 14 del CMCT.

Hoy es conveniente y oportuno que la comunidad técnica y científica abocada al tabaquismo acuerde y proponga estrategias de acción individual para los fumadores. Las mismas deben considerar no sólo la evidencia científica disponible, también la accesibilidad y equidad para que permitan romper el infame y complejo circuito de adicción-pobreza-enfermedad, donde las poblaciones de menores recursos y educación son las más afectadas. ${ }^{22}$ Necesitamos entrenar al personal de salud para que en cada una de sus actividades cotidianas puedan intervenir en estas poblaciones y ayuden a modificar su destino, determinado en parte por nacer en un país pobre, en un grupo social desaventajado y en el seno de una familia de pocos recursos.

Con las herramientas disponibles, comunitarias e individuales, las estrategias globales deberían establecerse con objetivos normativos para el descenso de la prevalencia de tabaquismo para los siguientes 10 años y también definir en forma dinámica y estratégica las acciones adecuadas para cada país o comunidad de Latinoamérica.

Las acciones de intervención deben plantearse con recomendaciones supranacionales de consenso, basadas en evidencias científicas, con estándares elevados y sin costo para las poblaciones vulnerables y de mayor consumo, exigiendo la asignación de recursos para alcanzar los objetivos propuestos. Así, los cuadros técnicos deberían diseñar estrategias de la misma manera que lo hacen para programas de vacunación, combate a la tuberculosis o malaria y exigir a los cuadros políticos ponerlas en la agenda para obtener los recursos necesarios, pero además deben percibir la necesidad de construir consenso de tales necesidades y transformarlas en políticas públicas. Es imperativo, entonces, que ambas corrientes de acción perciban la necesidad de evitar preconceptos, romper las barreras institucionales e ideológicas, evitar conflictos y confrontaciones y trabajar mancomunadamente en pos del aprovechamiento de todas las oportunidades para modificar las predicciones. Sería entonces, y sólo 
entonces, cuando podríamos decir que estaremos cambiando el curso de la epidemia de tabaquismo en nuestra región.

\section{Declaración de conflicto de intereses}

Declaramos no tener conflicto de intereses.

\section{Referencias}

I. Jha P, Chaloupka F. Curbing the Epidemic Governments and the Economics of Tobacco Control. Washington, DC:The International Bank for Reconstruction and Development/The World Bank, 1999 2. Peto R, Lopez A, Boreham J, MT, CJ H. Mortality from smoking in developed countries 1950-2000: indirect estimates from national vital statistics. Oxfoerd: Oxford University Press, 1994.

3. Ministry of Health. ABC Smoking Cessation Approach. 2007 [consultado: 2010 febrero 25]. Disponible en: https://smokingcessationabc.org.nz/. 4. Peto R, Darby S, Deo H, Silcocks P,Whitley E, Doll R. Smoking, smoking cessation, and lung cancer in the UK since 1950: combination of national statistics with two case-control studies. BMJ 2000;32I (7257):323-329. 5. Chapman S, MacKenzie R. The Global Research Neglect of Unassisted Smoking Cessation: Causes and Consequences. PLoS Med 2010;7(2): : 1000216.

6. West R, Stapleton J. Clinical and public health significance of treatments to aid smoking cessation. European Respiratory Review 2008; 17(II0):199-204.

7. Britton J. In defence of helping people to stop smoking. Lancet 2009;373:703-705.

8. Hatsukami D, Stead L, Gupta P.Tobacco Adiction. Lancet 2008;37I: 2027-2038.

9. Smith D. The historical decline of tobacco smoking among United States physicians: 1949-1984. Tob Induc Dis 2008;4(I):9.

10. Smith D, Leggat P.An international review of tobacco smoking in the medical profession: 1974-2004. BMC Public Health 2007;7(I):I 15.

II. Minervini M, Zabert G, Rondelli M, Gomez M, Castaño C, Buist A, et al. Tobacco Use among Argentine Physicians. Revista Argentina de Medicina Respiratoria 2006;3:100-105.

12. Chatkin J, Chatkin G. Learning about smoking during medical school: are we still missing opportunities? Int J Tuberc Lung Dis 2009;13(4):429-437.
13. World Health Organization. Framework Convention on Tobacco Control. Geneva:WHO, 2003.

14. World Health Organization. MPOWER: un plan de medidas para hacer retroceder la epidemia de tabaquismo. Geneva:WHO, 2008.

15. Müller F,Wehbe L. Smoking and smoking cessation in Latin America: a review of the current situation and available treatments. Int J Chron Obstruct Pulmon Dis 2008;3(2):285-293.

16. Zabert G. Los médicos y el tabaco. In: Müller F, ed. Cesación tabáquica: tiempo de intervenir. Buenos Aires: Editorial Polemos, 2006: I27-I48. 17. Silagy C, Lancaster T, Stead L, Mant D, Fowler G. Nicotine replacement therapy for smoking cessation (Cochrane Review) The Cochrane Library,Oxford: Update Software 2004(4).

18. Hughes J, Stead L, Lancaster T.Antidepressants for smoking cessation (Cochrane Review).The Cochrane Library,Oxford: Update Software 2004(4).

19. Cahill K, Stead L, Lancaster T. Nicotine receptor partial agonists for smoking cessation. (Cochrane Review). The Cochrane Library, Oxford Update Software (Cochrane Review). 2007(I).

20. Lasterto L. La pobreza en América Latina, Hay quienes viven con un dólar. 17 de agosto, 2003. Univision.com [consultado: 2010 marzo 16]. Disponible en: www.univision.com/content/content.jhtml.

21. Zamboni M, Lopez M, Zabert G. Equidad para el tratamiento del tabaquismo: nuevo desafío para modificar las predicciones de las consecuencias del tabaquismo en Latinoamérica. Prev Tab 2009; I (2):58-6I. 22. Reddy-Jacobs C, Téllez-Rojo M, Meneses-González F, CampuzanoRincón J, Hernández-Ávila. M. Pobreza, jóvenes y consumo de tabaco en México. Salud Publica Mex 2006;48(suppl I):S83-S90.

23. Carni J, Farre M. Drug Addiction. N Engl J Med 2003;349:975-986.

24. Raw M, Regan S, Rigotti N, McNeill A. A survey of tobacco dependence treatment services in 36 countries. Addiction 2009;104(2):279-287. 25. Sáenz de Miera-Juárez B, Jiménez-Ruiz JA, Reynales-Shigematsu LM, Lazcano-Ponce E, Hernández-Ávila M. El consumo de tabaco en los hogares mexicanos, 1994-2005. Salud Pública México 2007;49(suppl 2):S263-S269.

26. Ferrante D, Levy D, Peruga A, Compton C, Romano E. The role of public policies in reducing smoking prevalence and deaths: the Argentina tobacco policy simulation model. Rev Panam Salud Pública / Pan Am J Public Health 2007;2I(I):37-49.

27. Framework Convention Alliance. Countries' Ratification Status 2010 [consultado: 2010 febrero 25]. Disponible en: www.fctc.org/index. php?option=com_sobi2\&catid $=6 \&$ Itemid $=26$. 